Ciência Florestal, Santa Maria, v. 24, n. 4, p. 821-831, out.-dez., 2014

ISSN 0103-9954

\title{
FITOSSOCIOLOGIA DE FRAGMENTOS DE FLORESTA ESTACIONAL DECIDUAL NO ESTADO DE SANTA CATARINA - BRASIL
}

\author{
PHYTOSOCIOLOGY OF SEASONAL DECIDUOUS FOREST FRAGMENTS IN SANTA CATARINA \\ STATE - BRAZIL
}

\begin{abstract}
Lauri Amândio Schorn ${ }^{1}$ Leila Meyer ${ }^{2}$ Lucia Sevegnani ${ }^{3}$ Alexander Christian Vibrans ${ }^{1}$ Débora Vanessa $^{6}$ Lingner André Luis de Gasper ${ }^{4}$ Alexandre Uhlmann ${ }^{5}$ Márcio Verdi ${ }^{7}$ Anita Stival-Santos ${ }^{7}$
\end{abstract}

\section{RESUMO}

O estudo objetivou avaliar a fitossociologia da Floresta Estacional Decidual no Estado de Santa Catarina, em seus componentes arbóreo/arbustivo e regeneração natural, bem como comparar estes quanto à composição florística e inferir sobre o estádio de conservação dos remanescentes. Foram usados dados de 78 unidades amostrais levantadas no Inventário Florístico Florestal do Estado de Santa Catarina. Em cada unidade amostral foram amostrados os componentes arbóreo/arbustivo (DAP $\geq 10 \mathrm{~cm}$ ) em $4000 \mathrm{~m}^{2} \mathrm{e}$ a regeneração natural (altura $\geq 1,50 \mathrm{~m} ; \mathrm{DAP}<10 \mathrm{~cm}$ ) em $100 \mathrm{~m}^{2}$. Foi efetuado o cálculo dos parâmetros fitossociológicos e índices de diversidade de Shannon e equabilidade, bem como, classificaram-se as espécies quanto ao grupo ecológico. Para verificar a associação entre os dois componentes executou-se uma análise de ordenação NMSD (Non-metric Multidimensional Scaling) seguida do Teste de Mantel. Foram amostradas 245 espécies, sendo 215 no componente arbóreo/arbustivo e 79 exclusivas deste, 165 na regeneração natural e 30 exclusivas deste e, 136 comuns aos dois componentes. A NMDS apresentou uma tendência de associação entre as matrizes dos dois componentes, o que foi confirmado pelo Teste de Mantel $(\mathrm{r}=0,34 ; \mathrm{p}=0,001)$. A composição florística, bem como as espécies com maiores valores de importância se constituíram predominantemente de espécies secundárias, fato que evidencia o histórico de exploração e degradação da Floresta Estacional Decidual no Estado.

Palavras-chave: componente arbóreo/arbustivo; estrutura; regeneração natural; sub-bosque.

\section{ABSTRACT}

This study aimed to evaluate the species composition and structure of Seasonal Deciduous Forest in the southern Brazilian state of Santa Catarina, including the tree/shrub component and the natural regeneration. We also compared the floristic composition between the components and discussed about the conservation condition of forests. The study was based on sample plots established by the Floristic and Forest Inventory

1 Engenheiro Florestal, Dr., Professor do Departamento de Engenharia Florestal, Universidade de Blumenau, Rua São Paulo, 3250, CEP 89030-080, Blumenau (SC), Brasil. 1schorn@furb.br

2 Bióloga, Mestranda em Biologia Vegetal, Universidade Federal de Minas Gerais, Inventário Florístico- Florestal de Santa Catarina, Universidade de Blumenau, Rua São Paulo, 3250, CEP 89030-080, Blumenau (SC), Brasil. leilameyer@gmail.com

3 Bióloga, Dra ${ }^{\text {. }, ~ I n v e n t a ́ r i o ~ F l o r i ́ s t i c o-F l o r e s t a l ~ d e ~ S a n t a ~ C a t a r i n a, ~ U n i v e r s i d a d e ~ d e ~ B l u m e n a u, ~ R u a ~ S a ̃ o ~ P a u l o, ~} 3250$, CEP 89030-080, Blumenau (SC), Brasil. sevegn@furb.br

4 Biólogo,Msc., Doutorando em Biologia Vegetal, Universidade Federal de Minas Gerais, Inventário FlorísticoFlorestal de Santa Catarina, Universidade de Blumenau, Rua São Paulo, 3250, CEP 89030-080, Blumenau (SC), Brasil. algasper@gmail.com

5 Biólogo, Dr., Pesquisador da Embrapa Florestas, Estrada da Ribeira, Km 111, CEP 83411-000, Colombo (PR). alexandre@cnpf.embrapa.br

6 Engenheira Florestal, Msc., Inventário Florístico-Florestal de Santa Catarina, Universidade de Blumenau, Rua São Paulo, 3250, CEP 89030-080, Blumenau (SC).deboravanessa.ef@gmail.com

7 Biólogo(a), Mestrando em Ecologia, Universidade Federal do Rio Grande do Sul, Inventário Florístico-Florestal de Santa Catarina, Universidade de Blumenau, Rua São Paulo, 3250, CEP 89030-080, Blumenau (SC), Brasil.

Recebido para publicação em 22/11/2012 e aceito em 6/05/2013

Ci. Fl., v. 24, n. 4, out.-dez., 2014 
of Santa Catarina state. Tree/shrub component was sampled by 78 plots placed systematically with 4,000 $\mathrm{m}^{2}$ of sample area each, were individuals with DBH (diameter at breast height) $\geq 10 \mathrm{~cm}$ was measured. Regeneration (DBH $<10 \mathrm{~cm}$ and height $>1.50 \mathrm{~m}$ ) was sampled in subplots of $100 \mathrm{~m}^{2}$ each. Structure and diversity of sample plots were analyzed in both components; species were classified by their ecologic group. To test the association between components was made a Non-metric Multidimensional Scaling (NMSD) and Mantel test. We sampled 245 species, among them 215 in the tree/shrub component (79 of them exclusive), 165 species in the natural regeneration (30 exclusive ones) and 136 species common to the two components. The association between components was confirmed with NMSD and Mantel test $(\mathrm{r}=$ $0.34 ; p=0.001)$. Species with major importance value were mostly early secondary species that emphasizes the consequences of long term exploitation and degradation of Seasonal Deciduous Forest in Santa Catarina. Keywords: tree/shrub component; structure; natural regeneration; understory.

\section{INTRODUÇÃO}

A Floresta Estacional Decidual, no estado de Santa Catarina, distribui-se pela bacia hidrográfica do rio Uruguai, sobretudo ao longo do seu curso principal e nas porções média e baixa de seus afluentes. Sua área original era de $7670 \mathrm{~km}^{2}$, ocorrendo preferencialmente em altitudes de 150 a $800 \mathrm{~m}$ e, ocasionalmente, em até $900 \mathrm{~m}$ (KLEIN, 1972; IBGE, 1991). Sua vegetação apresenta deciduidade, especialmente das espécies emergentes e constituintes do dossel da floresta (KLEIN, 1972; 1978; IBGE, 1991) e, esta, possivelmente, resulta da ação de fatores climáticos restritivos como a diminuição do fotoperíodo e baixas temperaturas, com frequência anual média de 5 a 10 dias de geadas durante o inverno (NIMER, 1990; IBGE, 1991; ALBERTI et al., 2011).

Quanto a sua origem, Bigarella (1964) sugere que a Floresta Estacional Decidual representa uma vegetação recente em Santa Catarina, posterior à ocupação dos campos e da Floresta Ombrófila Mista. Desta forma, esta vegetação é considerada um prolongamento das florestas da bacia do rio Paraná, vinda através da Província de Missiones Argentina (RAMBO, 1951; 1956; KLEIN, 1972; PENNINGTON et al., 2009).

A cobertura florestal desta região fitoecológica em Santa Catarina é estimada em $16,1 \%$, conforme Vibrans et al. (2012). Esta redução é consequência da abundância de espécies madeiráveis de alto valor econômico, bem como da aptidão agrícola dos solos. Muitas áreas antes cobertas por floresta foram desmatadas e convertidas em áreas agrícolas e pecuárias, especialmente após a segunda metade do século XX (KLEIN, 1972). Atualmente, as florestas restringem-se a fragmentos esparsos e isolados, com grande perda de diversidade
(ALDRICH e HAMRICK, 1998). Remanescentes florestais com até 50 hectares representam $89 \%$, enquanto que remanescentes com mais de 200 hectares são raros, de acordo com Vibrans et al. (2012).

Em Santa Catarina, os levantamentos florísticos cobrindo a área de abrangência desta região fitoecológica, bem como seu mapeamento fitogeográfico, foram publicados por Klein (1972; 1978) e Reitz et al. (1979) e, mais recentemente, de modo pontual, a avaliação da estrutura arbórea efetuada por Ruschel et al. (2003; 2005; 2009). No entanto, persiste como a região fitoecológica com menor conhecimento acerca de sua flora e vegetação no Estado. Um componente negligenciado é o da regeneração natural, que representa o conjunto de indivíduos que tem potencial para constituir o dossel futuro da floresta (SWAINE e HALL, 1988; WARD e PARKER, 1989). A composição do dossel é resultado de uma série de fatores, dentre eles a composição atual da floresta. Portanto, qualquer influência da estrutura da floresta atual sobre a regeneração será refletida em sua manutenção (WARD e PARKER, 1989; FELFILI, 1991).

Assim, diante da intensa ação antrópica que ameaça a manutenção da diversidade da Floresta Estacional Decidual em Santa Catarina e da carência de conhecimento amplo desta, objetivou-se neste estudo diagnosticar a composição e estrutura de remanescentes florestais em seus componentes arbóreo/arbustivo e regeneração natural, além do estado de conservação dos remanescentes florestais avaliados. Testou-se, ainda, a hipótese de existência de associação entre a composição florística da regeneração natural e do componente arbóreo/ arbustivo. 


\section{MATERIAL E MÉTODOS}

O Inventário Florístico Florestal de Santa Catarina (IFFSC) levantou 78 unidades amostrais (UA) em fragmentos de Floresta Estacional Decidual do Estado (Figura 1). Cada UA é um conglomerado com área de $4000 \mathrm{~m}^{2}$, constituído de quatro subunidades $20 \mathrm{~m} \times 50 \mathrm{~m}$, orientadas na direção dos pontos cardeais (N, S, L e W), distantes $30 \mathrm{~m}$ do ponto central da unidade. Nas subunidades levantou-se o componente arbóreo/arbustivo que compreendeu todos os indivíduos com diâmetro a altura do peito (DAP) $\geq 10 \mathrm{~cm}$, bem como todas as plantas mortas ainda em pé que atendessem ao critério de inclusão. Na porção final de cada subunidade foi implantada uma subparcela de $5 \mathrm{~m}$ x $5 \mathrm{~m}$, destinada ao levantamento da regeneração natural que englobou todos os indivíduos com altura $\geq 1,50 \mathrm{~m}$ e DAP $\leq 10 \mathrm{~cm}$ (VIBRANS et al., 2010). O componente arbóreo/arbustivo contou com uma área amostrada total de $284.018 \mathrm{~m}^{2}$ e a regeneração natural, de $6860 \mathrm{~m}^{2}$. Na UA 1619 no município de Ipira não foi levantada a regeneração natural em decorrência do adensamento do sub-bosque por
Chusquea sp. Portanto, para a regeneração natural foram avaliadas somente $77 \mathrm{UA}$.

A identificação do material botânico foi realizada em campo ou após coleta e herborização, mediante consulta à coleção do Herbário FURB e consulta aos especialistas. A classificação das famílias seguiu APG III (2009). As espécies exóticas mensuradas nas UA foram incluídas na contagem de espécies. As espécies foram classificadas quanto ao grupo ecológico em pioneira, secundária ou climácica, conforme classificação adotada pelo IFFSC que teve como base a Flora Ilustrada Catarinense (REITZ, 1965-2011) e Reitz et al. (1979).

Os parâmetros fitossociológicos foram calculados para o componente arbóreo/arbustivo e regeneração natural, conforme Müeller-Dombois e Ellenberg (1974), assim como estimados os índices de diversidade de Shannon e de equidade de Pielou para cada UA em ambos os componentes, por meio do software Mata Nativa 2 (CIENTEC, 2006).

Para comparar a composição florística do componente arbóreo/arbustivo com a regeneração natural foi confeccionada uma matriz binária

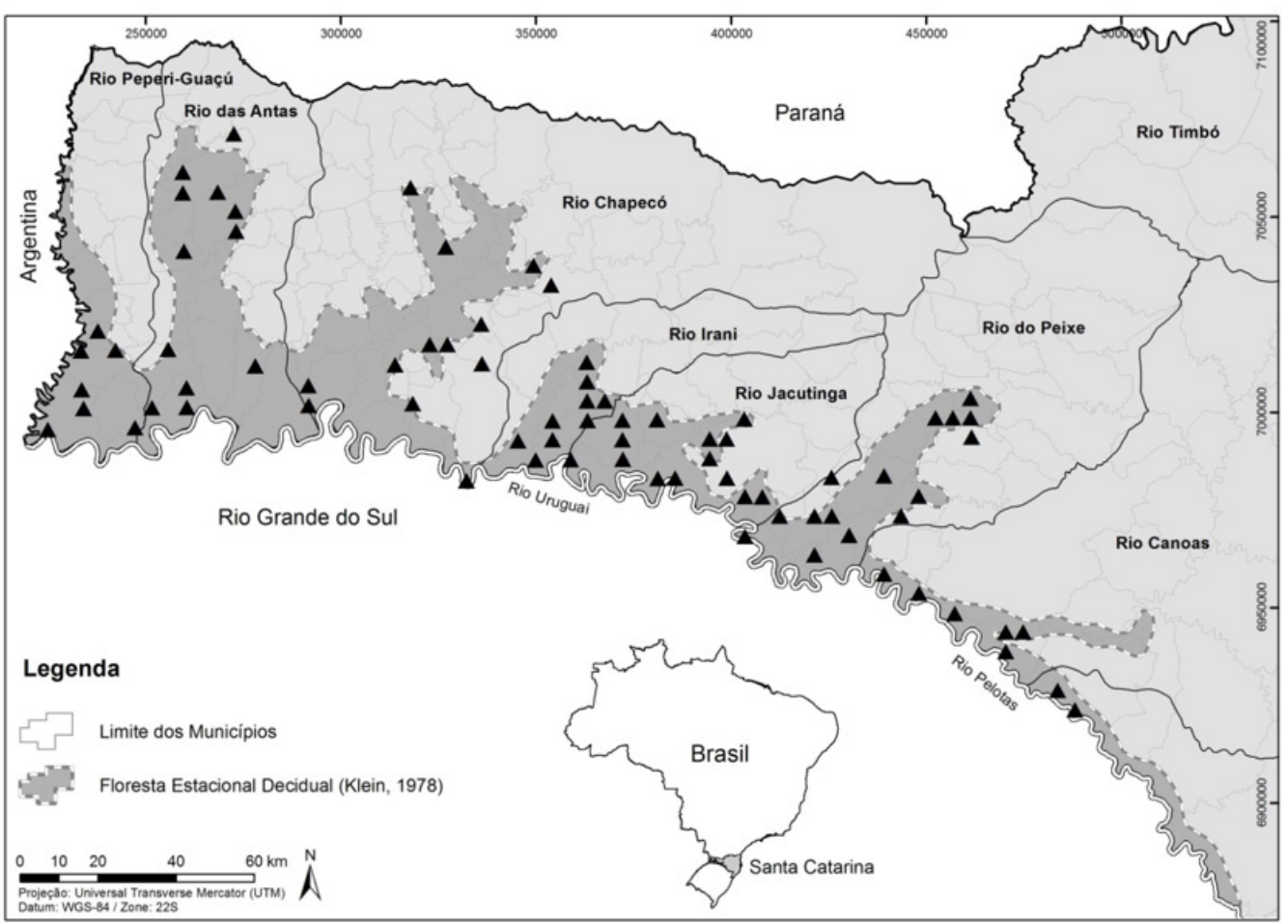

FIGURA 1: Localização das 78 unidades amostrais em fragmentos de Floresta Estacional Decidual no estado de Santa Catarina, Brasil.

FIGURE 1: Localization of the 78 sampling units in fragments Seasonal Deciduous Forest in Santa Catarina state, Brazil. 
com 154 linhas (UA) e 265 colunas (espécies). Nesta matriz, as espécies raras (aqui consideradas aquelas com menos de cinco registros), exóticas e não identificadas plenamente foram removidas, restando 132 espécies. Esta matriz foi submetida à Non-metric Multidimensional Scaling (NMDS), empregando o coeficiente de similaridade de Sorensen e teste de Monte Carlo com 999 permutações (MCCUNE e GRACE, 2002). Para testar a associação entre os dois componentes foram montadas duas matrizes binárias, cada uma composta por 77 linhas (UA de cada componente) e por 132 colunas (espécies), as quais foram submetidas ao Teste de Mantel, empregando o coeficiente de similaridade de Sorensen e teste de Monte Carlo com 999 permutações (MCCUNE e GRACE, 2002). Estas análises foram executadas no PC-ORD 6 (MCCUNE e MEFFORD, 2011).

\section{RESULTADOS E DISCUSSÃO}

Foram amostrados no componente arbóreo/arbustivo, 12.899 indivíduos, além de 824 plantas mortas ainda em pé, sendo três espécies de pteridófitas - Alsophila setosa Kaulf, Cyathea corcovadensis (Raddi) Domin e Dicksonia sellowiana Hook, uma gimnosperma - Araucaria angustifolia (Bertol.) Kuntze e 211 angiospermas, distribuídas em 140 gêneros e 62 famílias. Na regeneração natural foram 3325 indivíduos, sendo uma espécie de gimnosperma Araucaria angustifolia e 164 angiospermas, distribuídos em 111 gêneros e 48 famílias. No conjunto dos componentes foram amostradas 245 espécies, o que representa $35,4 \%$ das angiospermas citadas para as Florestas Estacionais Deciduais do Brasil por Stehmann et al. (2009). Foram encontradas três espécies ameaçadas de extinção de acordo com MMA (2008), sendo: Araucaria angustifolia, Dicksonia sellowiana e Ocotea odorifera (Vell.) Rohwer. $\mathrm{Na}$ amostra também estão presentes cinco espécies exóticas: Citrus limon (L.) Osbeck, Citrus reticulata Blanco, Hovenia dulcis Thunb, Morus nigra L. e Persea americana Mill., as quais foram incluídas na contagem de espécies.

As plantas mortas e ainda em pé representaram $6,4 \%$ do total de indivíduos amostrados. Em Floresta Ombrófila Densa no Rio de Janeiro, Silva e Nascimento (2001) registraram $9,8 \%$ de plantas mortas do total de indivíduos amostrados e sugeriram que tal mortalidade poderia estar associada com as perturbações sofridas. A
Floresta Estacional Decidual em Santa Catarina foi alvo de intensa supressão e fragmentação (KLEIN, 1972), processos que são apontados por Laurence et al. (2000) como intensificadores da mortalidade de plantas. Alguns fragmentos encontram-se em franco processo de sucessão ecológica, no entanto, continuam sofrendo perturbações antropogênicas. Vibrans et al. (2011) citaram que entre os fatores que podem estar influenciando a mortalidade nestas florestas estão: a exploração seletiva de madeira, roçada e/ou pastejo do gado no sub-bosque, caça, presença de espécies exóticas, instalação de trilhas, queimadas, bem como, a pressão exercida pelas práticas agrícola, pecuária, de mineração e a expansão imobiliária.

Tanto para o componente arbóreo/arbustivo quanto para a regeneração natural, as espécies secundárias representaram mais da metade do percentual do número de espécies e do número de indivíduos. No grupo das pioneiras, a regeneração natural apresentou maior porcentagem de espécies, mas, menor porcentagem de indivíduos quando comparado ao componente arbóreo/arbustivo. O inverso aconteceu para o grupo das climácicas (Figura 2). A predominância das espécies secundárias reflete o histórico de ocupação desta região fitoecológica no Estado. No início do século XX, sobretudo após a Guerra do Contestado, o oeste catarinense passou por um processo de ocupação efetivo por imigrantes majoritariamente europeus, resultando na exploração das florestas primárias pela indústria madeireira e/ou seu corte e substituição por agricultura e pecuária (KLEIN, 1978; ROSSETO, 1995; SILVA, 2010). Com a crescente migração da população rural em direção aos centros urbanos e, também, em virtude do Decreto ${ }^{\circ} 750 / 1993$ que proibiu a exploração da Mata Atlântica e posterior Lei $\mathrm{n}^{\mathrm{o}} 11.428 / 2006$ que definiu parâmetros de conservação e uso da vegetação do bioma Mata Atlântica (BRASIL, 1993; 2006), áreas florestais anteriormente exploradas ou mesmo áreas agrícolas e pastagens abandonadas puderam se recuperar. Estes fatos podem explicar a predominância das espécies secundárias e pioneiras nos fragmentos inventariados, onde ainda é constatada redução da cobertura florestal (SOS MATA ATLÂNTICA e INPE, 2012), mas em menor escala que aquela verificada antes da existência da legislação citada.

Os índices de diversidade de Shannon e equabilidade variaram entre 1,91 e 3,66 nats.ind ${ }^{-1} \mathrm{e}$ 0,64 e 0,96 para o componente arbóreo/arbustivo e, 0,51 e 3,05 nats.ind ${ }^{-1}$ e 0,28 a 0,98 para a regeneração 


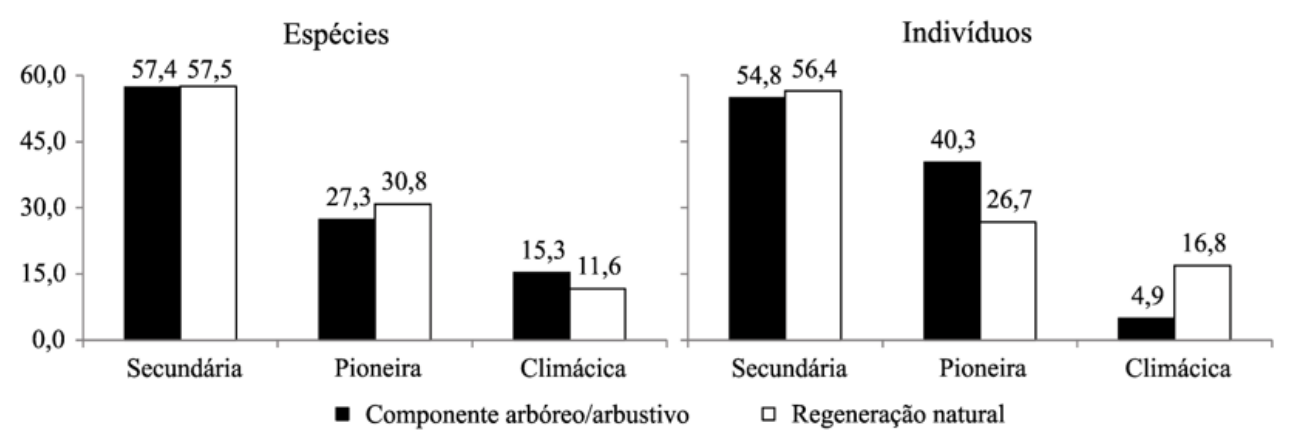

FIGURA 2: Distribuição do percentual do número de espécies e do número de indivíduos por grupo ecológico no componente arbóreo/arbustivo e na regeneração natural em fragmentos de Floresta Estacional Decidual no estado de Santa Catarina, Brasil.

FIGURE 2: Distribution of the percentage of the number of species and number of individuals per ecological group in component in the tree/shrub and natural regeneration in the Seasonal Deciduous Forest of Santa Catarina state.

natural, respectivamente (Tabela 1). No componente arbóreo/arbustivo, $60,3 \%$ das UA apresentaram valores para o índice de Shannon superiores a 3,00 nats.ind ${ }^{-1}$, enquanto que, na regeneração natural, apenas a UA 1188, localizada no município de Campos Novos, registrou índice superior a 3,00 nats. ind $^{-1}$. Comparativamente, no componente arbóreo/ arbustivo apenas o estudo de Ruschel et al. (2007) realizado no Parque Estadual do Turvo, RS, unidade de conservação com vegetação em bom estado de conservação, obteve valor superior aos registrados no presente estudo e, na regeneração natural, o de Scipioni et al. (2009), considerando os diferentes critérios de amostragem (Tabela 1).

No componente arbóreo/arbustivo, a densidade absoluta média foi de 490,1 ind.ha ${ }^{-1} \mathrm{e}$ a área basal média de $21,5 \mathrm{~m}^{2} \cdot \mathrm{ha}^{-1}$. Estes valores foram menores que os encontrados por Jarenkow e Waechter (2001) e Ruschel et al. (2007). Porém, os autores incluíram na amostra todos os indivíduos

TABELA 1: Levantamentos fitossociológicos do componente arbóreo/arbustivo e da regeneração natural para o Sul do Brasil. RF: região fitoecológica; S: riqueza; DA: densidade absoluta (ind.ha-1 ${ }^{-1}$; $\mathrm{AB}$ : área basal $\left(\mathrm{m}^{2} \cdot \mathrm{ha}^{-1}\right) ; \mathrm{H}^{\prime}$ : índice de Shannon; J: índice de equabilidade; FE: Florestas Estacionais; ${ }^{1}$ Amostragem em três transectos com $750 \mathrm{~m}$ cada.

TABLE 1: Phytosociology studies of tree/shrub component and natural regeneration in southern Brazilian. RF: phyto-ecological region; S: richness; DA: absolute density (ind.ha' ${ }^{-1}$ ); AB: basal area $\left(\mathrm{m}^{2} \cdot \mathrm{ha}^{-1}\right)$; H': Shannon index; J: equitability index; FE: Seasonal Forest; ${ }^{1}$ Sampling three transects with $750 \mathrm{~m}$ each.

\begin{tabular}{|c|c|c|c|c|c|c|c|c|}
\hline Autores & $\mathrm{RF}$ & Área & Amostragem & S & DA & $\mathrm{AB}$ & $\mathrm{H}^{\prime}$ & $\mathrm{J}$ \\
\hline \multicolumn{9}{|c|}{ Componente arbóreo/arbustivo } \\
\hline Farias et al. (1994) & $\mathrm{FE}$ & 0,75 & $\mathrm{DAP} \geq 9,5 \mathrm{~cm}$ & 51 & 400 & - & - & - \\
\hline $\begin{array}{l}\text { Jarenkow e Waechter } \\
\qquad(2001)\end{array}$ & $\mathrm{FE}$ & 1,00 & $\mathrm{DAP} \geq 5,0 \mathrm{~cm}$ & 55 & 1.855 & - & 2,24 & 0,56 \\
\hline Ruschel et al. (2007) & $\mathrm{FE}$ & 1 & $\mathrm{DAP} \geq 5,0 \mathrm{~cm}$ & 78 & 879 & 25,12 & 3,73 & 0,86 \\
\hline Presente estudo & FE & 28,40 & $\mathrm{DAP} \geq 10,0 \mathrm{~cm}$ & 215 & 490 & 21,47 & 1,91 a 3,66 & 0,64 a 0,96 \\
\hline \multicolumn{9}{|c|}{ Regeneração natural } \\
\hline Longhi et al. (2000) & $\mathrm{FE}$ & 0,02 & $\mathrm{H} \geq 10,0 \mathrm{~cm} ; \mathrm{DAP}<9,5 \mathrm{~cm}$ & 64 & 40.250 & - & - & - \\
\hline Wedy (2007) & $\mathrm{FE}$ & 0,01 & $\mathrm{H} \geq 0,2 \mathrm{~m}<1,0 \mathrm{~m}$ & 32 & 20.666 & - & 2,32 & 0,67 \\
\hline Scipioni et al. (2009) & FE & 0,28 & $\mathrm{DAP} \geq 1,6 \mathrm{~cm}<9,5 \mathrm{~cm}$ & 59 & 2.485 & - & 3,21 & 0,78 \\
\hline Leyser et al.(2012) & FE & 0,20 & $\mathrm{H} \geq 0,3 \mathrm{~cm} ; \mathrm{DAS}<4,7 \mathrm{~cm}$ & 64 & 8.245 & - & - & 0,68 \\
\hline Presente estudo & FE & 0,69 & $\mathrm{H} \geq 1,5 \mathrm{~m} ; \mathrm{DAP}<10,0 \mathrm{~cm}$ & 165 & 4.919 & - & 0,51 a 3,05 & 0,28 a 0,98 \\
\hline
\end{tabular}


com DAP $\geq 5 \mathrm{~cm}$. No trabalho de Farias et al. (1994), com inclusão dos indivíduos com $\mathrm{DAP} \geq 9,50 \mathrm{~cm}$, a densidade foi de 400 ind.ha $^{-1}$ (Tabela 1).

As espécies que se destacaram no componente arbóreo/arbustivo pelos elevados valores de densidade, frequência e dominância foram: Ocotea puberula (Rich.) Nees, Nectandra megapotamica (Spreng.) Mez, Luehea divaricata Mart. \& Zucc., pela densidade e frequência; Cupania vernalis Cambess. e Syagrus romanzoffiana (Cham.) Glassman pela frequência; pela densidade e dominância Nectandra lanceolata Nees (Tabela 2). As 20 espécies com maior valor de importância englobaram outras espécies conspícuas da Floresta Estacional Decidual do Estado, conforme Klein (1972; 1978): Cabralea canjerana (Vell.) Mart, Cedrela fissilis Vell., Chrysophyllum marginatum (Hook. \&Arn.) Radlk., Cordia americana (L.)
Gottschling \& J.S.Mill., Lonchocarpus campestris Mart. Ex Benth., Machaerium stipitatum (DC.) Vogel, Matayba elaeagnoides Radlk., Myrocarpus frondosus Allemão, Parapiptadenia rigida (Benth.) Brenan, Prunus myrtifolia (L.) Urb. e Trichilia claussenii C.DC. (Tabela 2). Hovenia dulcis, espécie exótica originaria da China e do Japão e, atualmente muito difundida no Sul do Brasil para obtenção de madeira e ornamentação (SOARES, 1972; CARVALHO, 1994; LORENZI et al., 2003), está entre as espécies com maior valor de importância neste componente.

Na regeneração natural, a densidade absoluta média foi de 4919 ind.ha $^{-1}$, valor que ficou abaixo dos encontrados nos demais estudos listados na Tabela 1, com exceção do trabalho de Scipioni et al. (2009). No entanto, há de se considerar os critérios de amostragem diferenciados entre os estudos. As

TABELA 2: Parâmetros fitossociológicos das 20 espécies com maior valor de importância no componente arbóreo/arbustivo em fragmentos de Floresta Estacional Decidual em Santa Catarina, Brasil.

TABLE 2: Phytosociology parameters of the 20 species with higher importance value in tree/shrub component of the Seasonal Deciduous Forest in Santa Catarina state, Brazil.

\begin{tabular}{|c|c|c|c|c|c|c|c|}
\hline Espécie & DA & DR & FA & FR & DoA & DoR & VI $(\%)$ \\
\hline Ocotea puberula (Rich.) Nees & 35,2 & 7,2 & 83,3 & 2,2 & 1,9 & 9,0 & 6,1 \\
\hline Nectandra megapotamica (Spreng.) Mez ${ }^{1}$ & 32,8 & 6,7 & 92,3 & 2,4 & 2,0 & 9,2 & 6,1 \\
\hline Luehea divaricata Mart. \& Zucc. ${ }^{1}$ & 27,9 & 5,7 & 87,2 & 2,3 & 1,6 & 7,3 & 5,1 \\
\hline Plantas mortas & 29,4 & 6,0 & 96,2 & 2,5 & 1,4 & 6,7 & 5,1 \\
\hline Nectandra lanceolata Nees & 19,4 & 4,0 & 74,4 & 1,9 & 1,4 & 6,4 & 4,1 \\
\hline Cupania vernalis Cambess. ${ }^{1}$ & 20,1 & 4,1 & 82,1 & 2,1 & 0,4 & 2,1 & 2,8 \\
\hline Machaerium stipitatum (DC.) Vogel $^{1}$ & 16,0 & 3,3 & 75,6 & 2,0 & 0,5 & 2,1 & 2,5 \\
\hline Syagrus romanzoffiana (Cham.) Glassman & 13,0 & 2,7 & 82,1 & 2,1 & 0,4 & 2,0 & 2,3 \\
\hline Cedrela fissilis Vell. & 10,9 & 2,2 & 76,9 & 2,0 & 0,4 & 2,1 & 2,1 \\
\hline Casearia sylvestris Sw. ${ }^{1}$ & 11,9 & 2,4 & 66,7 & 1,7 & 0,3 & 1,3 & 1,8 \\
\hline Parapiptadenia rigida (Benth.) Brenan ${ }^{1}$ & 6,3 & 1,3 & 52,6 & 1,4 & 0,6 & 2,8 & 1,8 \\
\hline Hovenia dulcis Thunb. & 12,0 & 2,5 & 43,6 & 1,1 & 0,4 & 1,7 & 1,8 \\
\hline Myrocarpus frondosus Allemão & 6,8 & 1,4 & 61,5 & 1,6 & 0,3 & 1,5 & 1,5 \\
\hline Chrysophyllum marginatum (Hook. \& Arn.) Radlk. & 6,3 & 1,3 & 52,6 & 1,4 & 0,4 & 1,8 & 1,5 \\
\hline Matayba elaeagnoides Radlk. & 9,1 & 1,9 & 48,7 & 1,3 & 0,3 & 1,2 & 1,5 \\
\hline Cabralea canjerana (Vell.) Mart. & 6,4 & 1,3 & 55,1 & 1,4 & 0,3 & 1,5 & 1,4 \\
\hline Lonchocarpus campestris Mart. ex Benth. ${ }^{1}$ & 6,5 & 1,3 & 65,4 & 1,7 & 0,3 & 1,2 & 1,4 \\
\hline Trichilia claussenii C.DC. ${ }^{1}$ & 8,5 & 1,7 & 53,9 & 1,4 & 0,2 & 0,9 & 1,4 \\
\hline Cordia americana (L.) Gottschling \& J.S.Mill. & 3,8 & 0,8 & 50,0 & 1,3 & 0,4 & 1,8 & 1,3 \\
\hline Prunus myrtifolia (L.) Urb. & 5,5 & 1,1 & 51,3 & 1,3 & 0,3 & 1,4 & 1,3 \\
\hline Demais espécies (195) & 182,0 & 37,2 & 2287,0 & 59,5 & 7,1 & 33,1 & 43,3 \\
\hline Total & 490,1 & 100,0 & 3834,6 & 100,0 & 21,5 & 100,0 & 100,0 \\
\hline
\end{tabular}

Em que: DA: densidade absoluta (ind.ha $\left.{ }^{-1}\right)$; DR: densidade relativa (\%); FA: frequência absoluta (\%); FR: frequência relativa $(\%)$; DoA: dominância absoluta $\left(\mathrm{m}^{2} . \mathrm{ha}^{-1}\right)$; DR: dominância relativa (\%); VI: valor de importância $(\%)$. ${ }^{1}$ Espécie com maior valor de importância comum na regeneração natural. 
espécies que registraram altos valores de densidade e frequência foram: Actinostemon concolor (Spreng.) Müll. Arg., Cupania vernalis, Trichilia elegans A. Juss. e Lonchocarpus campestris Mart. ex Benth; densidade Myrcia oblongata DC. e Pilocarpus pennatifolius Lem.; e frequência: Nectandra megapotamica (Tabela 3). Dentre as 20 espécies com maior valor de importância destacaram-se espécies marcantes desta região fitoecológica, majoritariamente arbustos e arvoretas que compõem o sub-bosque ou subdossel da floresta, conforme Klein (1972; 1978): Allophylus edulis (A.St.-Hil. et al.) Hieron. ex Niederl., Helietta apiculata Benth., Pilocarpus pennatifolius, Sorocea bonplandii (Baill.) W.C. Burger et al. e Urera baccifera (Baill.) W.C. Burger et al. (Tabela 3).

Oito foram as espécies com maior valor de importância comuns entre os dois componentes:
Casearia sylvestris, Cupania vernalis, Luehea divaricata, Machaerium stipitatum, Nectandra megapotamica, Parapiptadenia rigida, Lonchocarpus campestris e Trichilia claussenii, o que denota uma tendência de manutenção das espécies que atualmente possuem maior valor de importância no componente arbóreo/arbustivo (Tabelas 2 e 3).

Comparando as espécies amostradas nos dois componentes, 79 (32,2\%) espécies foram encontradas exclusivamente no componente arbóreo/arbustivo, 30 (12,3\%) foram exclusivas da regeneração natural e, $136(55,5 \%)$ foram comuns a ambos. Dentre as espécies exclusivas da regeneração natural apenas Myrsine balansae (Mez) Arechav. possui hábito arbóreo, sendo as demais arvoretas ou arbustos. Dentre as 79 espécies exclusivas do componente arbóreo/arbustivo estão: Ateleia

TABELA 3: Parâmetros fitossociológicos das 20 espécies com maior valor de importância na regeneração natural em fragmentos de Floresta Estacional Decidual em Santa Catarina, Brasil.

TABLE 3: Phytosociology parameters of the 20 species with higher importance value in natural regeneration of the Seasonal Deciduous Forest in Santa Catarina state, Brazil.

\begin{tabular}{|c|c|c|c|c|c|}
\hline Espécie & DA & DR & FA & FR & VI $(\%)$ \\
\hline Actinostemon concolor (Spreng.) Müll. Arg. & 402,4 & 8,2 & 55,8 & 3,7 & 4,0 \\
\hline Cupania vernalis Cambess. ${ }^{1}$ & 233,7 & 4,8 & 58,4 & 3,9 & 2,9 \\
\hline Trichilia elegans A.Juss. & 220,4 & 4,5 & 52,0 & 3,4 & 2,6 \\
\hline Lonchocarpus campestris Mart. ex Benth. ${ }^{1}$ & 238,2 & 4,8 & 39,0 & 2,6 & 2,5 \\
\hline Nectandra megapotamica (Spreng.) Mez & 133,1 & 2,7 & 49,4 & 3,3 & 2,0 \\
\hline Pilocarpus pennatifolius Lem. & 186,4 & 3,8 & 32,5 & 2,1 & 2,0 \\
\hline Machaerium stipitatum (DC.) Vogel ${ }^{1}$ & 137,6 & 2,8 & 29,9 & 2,0 & 1,6 \\
\hline Casearia sylvestris Sw. ${ }^{1}$ & 119,8 & 2,4 & 31,2 & 2,1 & 1,5 \\
\hline Sorocea bonplandii (Baill.) W.C.Burger et al. & 108,0 & 2,2 & 32,5 & 2,1 & 1,5 \\
\hline Myrsine umbellata Mart. & 115,4 & 2,4 & 27,3 & 1,8 & 1,4 \\
\hline Urera baccifera (Baill.) W.C. Burger et al. & 90,2 & 1,8 & 33,8 & 2,2 & 1,4 \\
\hline Trichilia claussenii C.DC. ${ }^{1}$ & 79,9 & 1,6 & 33,8 & 2,2 & 1,3 \\
\hline Allophylus edulis (A.St.-Hil. et al.) Hieron. ex Niederl. & 91,7 & 1,9 & 27,3 & 1,8 & 1,2 \\
\hline Myrcia oblongata DC. & 162,7 & 3,3 & 2,6 & 0,2 & 1,2 \\
\hline Luehea divaricata Mart. \& Zucc. ${ }^{1}$ & 76,9 & 1,6 & 27,3 & 1,8 & 1,1 \\
\hline Sebastiania brasiliensis Spreng. & 68,0 & 1,4 & 28,6 & 1,9 & 1,1 \\
\hline Helietta apiculata Benth. & 99,1 & 2,0 & 14,3 & 0,9 & 1,0 \\
\hline Justicia brasiliana Roth & 62,1 & 1,3 & 24,7 & 1,6 & 1,0 \\
\hline Parapiptadenia rigida (Benth.) Brenan ${ }^{1}$ & 87,3 & 1,8 & 13,0 & 0,9 & 0,9 \\
\hline Strychnos brasiliensis Mart. & 57,7 & 1,2 & 20,8 & 1,4 & 0,9 \\
\hline Demais espécies (145) & 2147,9 & 43,6 & 881,9 & 58,4 & 34,1 \\
\hline Total & 4918,6 & 100,0 & 1515,6 & 100,0 & 100,0 \\
\hline
\end{tabular}

Em que: DA: densidade absoluta (ind.ha-1); DR: densidade relativa (\%); FA: frequência absoluta (\%); FR: frequência relativa (\%); VI: valor de importância (\%). ${ }^{1}$ Espécie com maior valor de importância comum ao componente arbóreo/ arbustivo. 
glazioveana Baill., Aralia warmingiana (Marchal) J.W en, Handroanthus heptaphyllus Mattos, Jacaranda puberula Cham., Peltophorum dubium (Spreng.) Taub., entre outras. O fato delas não terem sido amostradas entre os regenerantes pode estar relacionado às características ecológicas particulares de cada espécie, bem como a metodologia de amostragem empregada pode não ter favorecido o levantamento satisfatório de todas as espécies regenerantes. Ressalta-se que das 136 espécies comuns aos dois componentes, algumas foram encontradas com baixa densidade na regeneração natural, como Erythrina falcata amostrada com somente 1,48 ind.ha-1 ${ }^{-1}$ Apuleia leiocarpa (Vogel) J.F. Macbr. $(5,92)$, Ocotea pulchella (Nees\& Mart.) Mez $(4,44)$, Cordia trichotoma (Vell.) Arráb. ex Steud. $(8,88)$ e Ilex paraguariensis A.St.-Hil. $(10,36)$.

A análise de ordenação NMDS apresentou uma tendência de relação entre a composição do componente arbóreo/arbustivo com a regeneração natural (Figura 3), o que foi confirmado pelo Teste de Mantel, que acusou uma associação significativa entre as matrizes dos dois componentes $(r=0,34$; $\mathrm{p}=0,001)$.

De certo modo, tal relação entre os dois componentes é esperada, tendo em vista que a regeneração natural é resultado da dispersão de propágulos provenientes das espécies em estágio reprodutivo do componente arbóreo/arbustivo, ou ainda, pela reprodução vegetativa. Mas, resultado inverso poderia ser esperado, pois a amostragem da regeneração natural incluiu, além de indivíduos jovens das espécies de dossel e subdossel, as espécies conspícuas de sub-bosque. No estudo de Leyser et al. (2012), não foi verificada uma associação entre as matrizes de abundância nos componentes arbóreo regenerante e adulto, em um remanescente de Floresta Estacional Decidual, sugerindo que este padrão pode estar diretamente ligado a uma separação de nichos. Ressalta-se que estes autores utilizaram dados quantitativos enquanto que o presente estudo, dados qualitativos (presença ou ausência das espécies), e a abrangência de área pode facilitar a homogeneização das espécies, no sentido de serem amostradas em mais de uma unidade amostral.

Salles e Schiavini (2007) verificaram que, apesar da similaridade florística de $61 \%$ entre os componentes arbóreo e de regeneração natural, as espécies comuns não apresentaram a mesma ordenação em relação ao valor de importância, principalmente pela maior densidade das espécies comuns na regeneração natural. Para Swaine e Hall (1988), a composição futura do dossel da floresta é resultado, pelo menos em parte, dos indivíduos

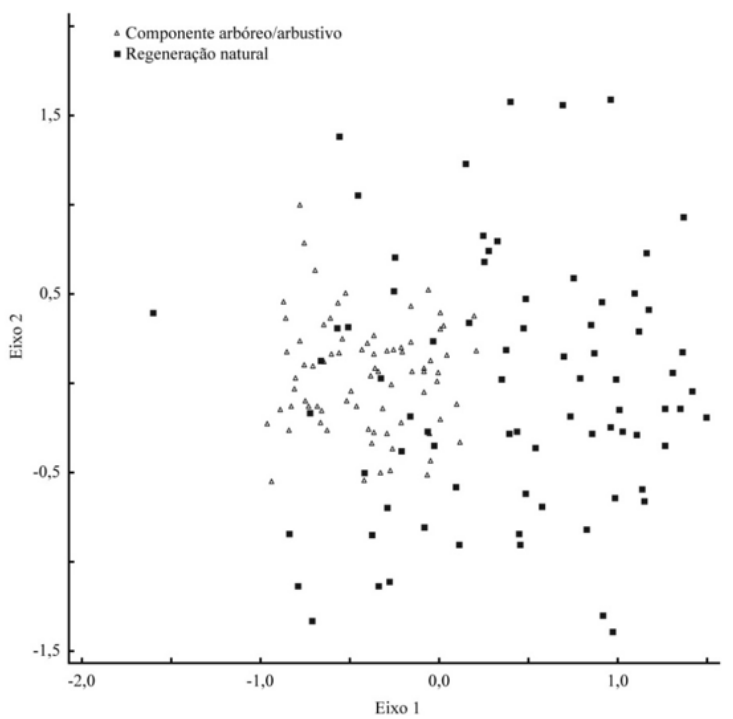

FIGURA 3: Resultado da Non-metric Multidimensional Scaling (NMDS) entre as unidades amostrais do componente arbóreo/arbustivo e regeneração natural em fragmentos de Floresta Estacional Decidual em Santa Catarina, Brasil.

FIGURE 3: Result of Non-metric Multidimensional Scaling (NMDS) between sample plots of the tree/ shrub component and natural regeneration in fragments of the Seasonal Deciduous Forest in Santa Catarina state, Brazil. 
jovens crescendo abaixo do dossel atual. No entanto, não é possível assumir com precisão que os indivíduos jovens atuais representarão o dossel futuro da floresta, pois cada espécie apresenta características próprias de fecundidade e de taxas de crescimento e mortalidade, fatores que podem alterar a composição dos juvenis ao longo do tempo, bem como, da floresta como um todo.

A repetição da composição florística e da dominância de algumas espécies muitas vezes está relacionada aos fatores de perturbação da floresta, natural ou antropogênicos, como a exploração seletiva de madeira que, por abrir clareiras, favorece espécies pioneiras e secundárias, o efeito de borda ampliado como resultado do pequeno tamanho dos fragmentos florestais (LAURANCE, 1998; 2002) e, ainda a possível redução dos agentes dispersores, resultante da caça, da perda de habitat e distância entre fragmentos bem conservados ricos em biodiversidade de plantas e animais (COSTA, 2012; DIRZO e MENDOZA, 2007).

\section{CONCLUSÕES}

Os fragmentos de Floresta Estacional Decidual estudados no estado de Santa Catarina apresentam significativa diversidade florística, com predomínio de espécies secundárias, seguidas de pioneiras e, em menor representatividade, das climácicas. Há uma tendência de manutenção das espécies encontradas com maior valor importância no componente arbóreo/arbustivo, em decorrência da atual densidade e frequência destas espécies na regeneração natural. Foi confirmada uma tendência à associação entre a composição da regeneração natural a do componente arbóreo/arbustivo. Mesmo assim, percebe-se uma perda da diversidade de espécies e da estrutura da floresta, associadas a fatores antrópicos.

\section{AGRADECIMENTOS}

À Fundação de Amparo à Pesquisa e Inovação do Estado de Santa Catarina (FAPESC) e ao Serviço Florestal Brasileiro (SFB) por financiarem o projeto Inventário Florístico Floresta de Santa Catariana. Aos proprietários das florestas amostradas e aos taxonomistas de diversas famílias botânicas pela identificação voluntária do material botânico.

\section{REFERÊNCIAS BIBLIOGRÁFICAS}

ALBERTI, L. F. et al. Padrão fenológico de árvores e a relação com o clima. In: SCHUMACHER, M. V. et al. (Eds.). A Floresta Estacional Subtropical: caracterização e ecologia no Rebordo do Planalto Meridional. Santa Maria: UFSM, 2011.

ALDRICH, P. R.; HAMRICK, J. L. Reproductive Dominance of Pasture Trees in a Fragmented Tropical Forest Mosaic. Science, New York, n. 281, p. 103-105, 1998.

APG III. An update of the Angiosperm Phylogeny Group classification for the orders and families of flowering plants: APG III. Botanical Journal of the Linnean Society, v. 161, n. 2, p. 105-121, 2009. BIGARELLA, J. J. Variações climáticas no Quaternário e suas implicações no revestimento florístico do Paraná. Boletim Paranaense de Geografia, Curitiba, n. 10/15, p. 211-231, 1964.

BRASIL. Decreto $\mathbf{n}^{\circ} \mathbf{7 5 0}$, de 10 de fevereiro de 1993. Disponível em <http://www.ibama.gov. br/flora/decretos/750_93.pdf $>$. Acesso em 7 de novembro de 2012.

BRASIL. Lei $\mathbf{n}^{0} \mathbf{1 1 . 4 2 8}$, de 22 de dezembro de 2006. Disponível em <http://www.planalto.gov.br/ ccivil 03/ ato2004-2006/2006/lei/111428.htm>. Acesso em 7 de novembro de 2012.

CARVALHO, P. E. R. Ecologia, silvicultura e usos da uva-do-Japão (HoveniadulcisThunberg). Colombo: EMBRAPA-CNPFlorestas, 1994.

CIENTEC. Software Mata Nativa 2: Sistema para análise fitossociológica, elaboração de inventários e planos de manejo de florestas nativas. Viçosa: Cientec, 2006.

COSTA, J. B. P. Reduced availability of large seeds constrains Atlantic forest regeneration. Acta Oecologica, Paris, n. 39, p. 61-66, 2012.

DIRZO, R.; MENDOZA, E. Size-related differential seed predation in a heavily defaunatedNeotropical Rain Forest. Biotropica, Zurich, n. 39, p. 355-362, 2007.

FARIAS, J. A. C. et al. Estrutura fitossociológica de uma Floresta Estacional Decidual na região de Santa Maria, RS. Ciência Florestal, Santa Maria, n. 4, p. 109-128, 1994.

FELFILI, J. M. Dynamics of the natural regeneration in the Gama gallery forest in central Brazil. Forest Ecologyand Management, Victoria, n. 91, p. 235-245, 1991.

IBGE. Manual técnico da vegetação brasileira. Rio de Janeiro: IBGE, 1991. 
JARENKOW, J.A.; WAECHTER, J. L. Composição, estrutura e relações florísticas do componente arbóreo de uma floresta estacional no Rio Grande do Sul, Brasil. Revista Brasileira de Botânica, São Paulo, n. 24, p. 263-272, 2001.

KLEIN, R. M. Árvores nativas da Floresta Subtropical do Alto Uruguai. Sellowia, Itajaí, n. 24, p. 9-62, 1972.

KLEIN, R. M. Mapa fitogeográfico de Santa Catarina.In: REITZ, R. (Ed.). Flora Ilustrada Catarinense. Itajaí: Herbário Barbosa Rodrigues, 1978.

LAURANCE, W. F. et al. Rain forest fragmentation and the dynamics of Amazonian tree communities. Ecology, New York, n. 79, p. 2032-2040, 1998.

LAURANCE, W. F. et al. Rainforest fragmentation kills big trees. Nature, London, n. 404, p. 836, 2000. LAURANCE, W. F. et al. Ecosystem decay of Amazonian forest fragments, a 22-year investigation.

Conservation Biology, Boston, n. 16, p. 605-618, 2002.

LEYSER, G. et al. Regeneração de espécies arbóreas e relações com componente adulto em uma floresta estacional no vale do rio Uruguai, Brasil. Acta Botanica Brasilica, Brasília, n. 26, p. 74-83, 2012.

LONGHI, S. J. et al. Aspectos fitossociológicos de fragmento de Floresta Estacional Decidual, Santa Maria, RS. Ciência Florestal, Santa Maria, n. 10, p. 59-74, 2000.

LORENZI, H. Árvores exóticas do Brasil: madeireiras, ornamentais e aromáticas. Nova Odessa: Plantarum, 2003.

MCCUNE, B. J.; GRACE. J. B. Analysis of Ecological Communities. Oregon: MJM, 2002.

MCCUNE, B. J.; MEFFORD, M. J. PC-ORD. Multivariate analysis of Ecological Data. Version 6.0, Oregon: MJM, 2011.

Brasil. Ministério do Meio Ambiente. Instrução normativa $n^{\circ} 6$, de 23 de setembro de 2008 . Disponível em <http://www.mma.gov.br/ estruturas/179/_arquivos/179_05122008033615. pdf $>$. Acessoem 7 de novembro de 2012.

MÜELLER-DOMBOIS, D.; ELLENBERG， $\mathrm{H}$. Aims and methods of vegetation ecology. New York: Wiley, 1974.

NIMER, E. Clima. In: IBGE. Geografia do Brasil: Região Sul. Rio de Janeiro: IBGE, 1990.

PENNINGTON, R. T. et al. Woody plant diversity, evolution, and ecology in the tropics: perspectives from Seasonally Dry Tropical Forests. Annual Review of Ecology, Evolution and Systematics,
California, n. 40, p. 437-457, 2009.

RAMBO, B. A. Imigração da selva higrófila no Rio Grande do Sul. Anais Botânicos, Itajaí, n. 3, p. 55-91, 1951.

RAMBO, B. A. Der Regenwald an oberen Uruguay. Sellowia, Itajaí, n. 7/8, n. 183-233, 1956.

REITZ, R. Flora Ilustrada Catarinense. Itajaí: Herbário Barbosa Rodrigues, 1965-2011.

REITZ, R. et al. Projeto Madeira - Santa Catarina. Florianópolis: Lunardelli, 1979.

ROSSETO, S. Síntese histórica da região oeste. In: CEOM. Centro de Organização da Memória Sociocultural do Oeste (Santa Catarina). Para uma história do oeste catarinense: 10 anos de CEOM. Chapecó: Ed. da UNOESC, 1995.

RUSCHEL, A. R. et al. Evolução do uso e valorização das espécies madeiráveis da Floresta Estacional Decidual do Alto-Uruguai, SC. Ciência Florestal, Santa Maria, n. 13, p. 153-166, 2003.

RUSCHEL, A. R. et al. Valuation and characterization of the timber species in remnants of the Alto Uruguay River ecosystem, southern Brazil. Forest Ecology and Management, Victoria, n. 217, p. 103-116, 2005.

RUSCHEL, A. R. et al. Woody plant species richness in the Turvo State Park, a large remnant of Deciduous Atlantic Forest, Brazil. Biodiversity and Conservation, New York, n. 16, p. 1699-1714, 2007.

RUSCHEL, A. R. et al. Estrutura e composição florística de dois fragmentos da Floresta Estacional Decidual do Alto-Uruguai, SC. Ciência Florestal, Santa Maria, n. 19, p. 225-236, 2009.

SALLES, J. G.; SCHIAVINI, I. Estrutura e composição do estrato de regeneração em um fragmento florestal urbano: implicações para a dinâmica e a conservação da comunidade arbórea. Acta Botanica Brasilica, Brasília, n. 21, p. 223-233, 2007.

SCIPIONI, M. C. et al. Regeneração natural de um fragmento da Floresta Estacional Decidual na reserva biológica do Ibicuí-Mirim (RS). Floresta, Curitiba, n. 39, p. 675-690, 2009.

SILVA, G. C.; NASCIMENTO, M. T. Fitossociologia de um remanescente de mata sobre tabuleiros no norte do estado do Rio de Janeiro (Mata do Carvão). Revista Brasileira de Botânica, São Paulo, n. 24, p. 51-62, 2001.

SILVA, A. L. Fazendo cidade: memória e urbanização no extremo oeste catarinense. Chapecó: Argos, 2010.

SOARES, M. A. F. Ramnáceas. In: REITZ, R. (Ed.). 
Flora Ilustrada Catarinense. Itajaí: Herbário Barbosa Rodrigues, 1972.

SOS Mata Atlântica \& INPE. Disponível em $\quad<$ http://www.sosma.org.br/index. content\&action $=$ contentDetails\&idContent $=1069>$. Acesso em 30 de junho de 2012.

STEHMANN, J. R. et al. Plantas da Floresta Atlântica. Rio de Janeiro: Instituto de Pesquisas Jardim Botânico do Rio de Janeiro, 2009.

SWAINE, M. D.; HALL, J. B. The mosaic theory of forest regeneration and the determination of forest composition in Ghana. Journal of Tropical Ecology, Cambridge, n. 4, p. 253-269, 1988.

VIBRANS, A. C. et al. Inventário florístico florestal de Santa Catarina (IFFSC): aspectos metodológicos e operacionais. Pesquisa Florestal Brasileira, Colombo, n. 30, p. 291-302, 2010.

VIBRANS, A. C. et al. Inventário Florístico
Florestal de Santa Catarina. Relatório final. Blumenau: Universidade Regional de Blumenau, 2011.

VIBRANS, A. C. et al. How much remains of the Brazilian Atlantic forest in the state of Santa Catarina? Assessing the accuracy of forest cover maps using ground data from the Santa Catarina Forest and Floristic Inventory. Remote Sensing of Environment, Amsterdam, 2012. (no prelo)

WARD, J. S.; PARKER, G.R. Spatial dispersion of woody regeneration in an old-growth forest. Ecology, New York, n. 70, p. 1279-1285, 1989.

WEDY, G. O. Estrutura e dinâmica da regeneração natural de espécies arbóreas na Floresta Estacional do Parque Estadual do Turvo, Derrubadas, Rio Grande do Sul. 55 f. Dissertação (Mestrado em Botânica) - Universidade Federal do Rio Grande do Sul, Porto Alegre, 2007. 